\title{
Las políticas de apoyo a las familias: ¿asignatura pendiente de las políticas sociales vascas? ${ }^{1}$
}

\section{SIIS Centro de Documentación y Estudios, Fundación Eguía-Careaga}

<estudios@siis.net>

\begin{abstract}
Artikulu honetan konparatzen da EAE, Estatu espainiarra eta gure inguruko herrialdeetako familiasostengurako politiketako gastua. Azterlanak azaltzen duenez, europarraren aldean baxua izaten jarraitzen du euskal familia-politiketan egindako gastu publikoak, eta haurtzaroko arreta-zerbitzuek baldintzatzen dute gehien, eta funtsean, familiakenkariak, eta horien lehenetsitako onuradunak izaten dira errenta ertain eta altuko taldeak. Halaber, bertan nabarmentzen da Euskadiko errenta ertaineko familiek babes urriagoa izaten dutela Europako herrialde gehienen aldean, eta horren ondorioz deribatzen -edo orekatzen- da presio fiskal eskasagoa. Errenta baxu edo diru-sarbide propiorik gabeko familien kasuan, aldiz, babes-maila antzekoa edo batzuetan hobea izaten dute Europako herrialdeen aldean, baina babes hori ez da jasotzen familia-prestazioetatik, eta heltzen da diru-sarbideak bermatzeko sistematik.
\end{abstract}

\section{GAKO-HITZAK:}

Pobrezia, familia-politikak, haurtzaroa, gizartegastua, prestazio ekonomikoak, azterlan konparatiboa.
Este artículo compara el gasto en políticas de apoyo a las familias de la CAPV y del Estado español con el de los países de nuestro entorno. El análisis muestra que el gasto vasco en políticas familiares sigue siendo bajo en relación al europeo y que está muy determinado por los servicios de atención infantil y, fundamentalmente, por las desgravaciones familiares, de las que se benefician, con preferencia, los grupos de rentas medias y altas. Además, se constata que las familias de rentas medias están en Euskadi mucho menos protegidas que en la mayor parte de los países europeos, lo que se deriva de -o se compensa por- una menor presión fiscal. Las familias de rentas bajas o sin ingresos propios, en cambio, gozan de una protección similar, o incluso mejor, que la existente en la mayor parte de Europa, pero dicha protección no se deriva de las prestaciones familiares, sino del sistema de garantía de ingresos.

\section{Palabras Clave:}

Política familiar, gasto social, ayudas a la familia, servicios de infancia, impuestos, País Vasco, Unión Europea. 


\section{Evolución del gasto social en la función familia e infancia en la UE y en Euskadi: se mantiene el diferencial con Europa}

En este artículo se comparan, desde el punto de vista del gasto, las políticas de apoyo a las familias de la Comunidad Autónoma del País Vasco (CAPV) y del Estado español en relación a las de los países de nuestro entorno. La fórmula más sencilla para evaluar el gasto social destinado a la infancia en los países de la UE consiste en analizar los datos que ofrece el Sistema Europeo de Estadísticas Integradas de la Protección Social (SEEPROS) de Eurostat, y compararlos con los que ofrece la Cuenta de la Protección Social que elabora Eustat para la CAPV, utilizando la misma metodología. Si bien es cierto que el SEEPROS plantea importantes limitaciones para el análisis del gasto social destinado a la infancia ${ }^{2}$, no debe olvidarse que constituye la principal fuente de datos europeos comparables en materia de protección social y que permite identificar, con toda nitidez, las tendencias evolutivas básicas y las principales diferencias entre los países de la UE. Los datos disponibles en el momento actual se limitan, para la CAPV, a 2010, es decir, al momento en el que empezaban a aplicarse en los países del sur de Europa las primeras medidas encaminadas a la reducción del déficit público.
El Gráfico 1 pone de manifiesto, en primer lugar, la evolución del gasto en relación al PIB en la función familia e hijos para la UE15 y para la CAPV entre 1995 y $2010^{3}$. Como se observa en la tabla, pese al crecimiento continuo del gasto en esta función en la CAPV, el diferencial con la UE sigue siendo elevado y se reduce muy ligeramente a lo largo del tiempo. También es importante señalar que entre 2009 y 2010 se produjo una caída en el nivel de gasto europeo, y una desaceleración en el caso vasco, particularmente notable si se tiene en cuenta que también el PIB cayó, o creció en muy escasa medida, en esos años.

El Gráfico 2 muestra el diferencial entre la UE y la CAPV para tres indicadores diferentes: el gasto en familia e infancia en relación al PIB, el gasto en el conjunto de las prestaciones sociales en relación al PIB y el gasto en familia e infancia en relación al conjunto de las prestaciones sociales.

Los datos muestran que el diferencial en relación a la UE, si consideramos el conjunto del gasto en protección social, es relativamente pequeño (en 2010, el gasto vasco equivalía al $83 \%$ del europeo) y que, además, se ha reducido desde el inicio de la crisis, debido a la reducción del PIB y el incremento en el gasto social como consecuencia de aquélla (fundamentalmente debido a las prestaciones de garantía

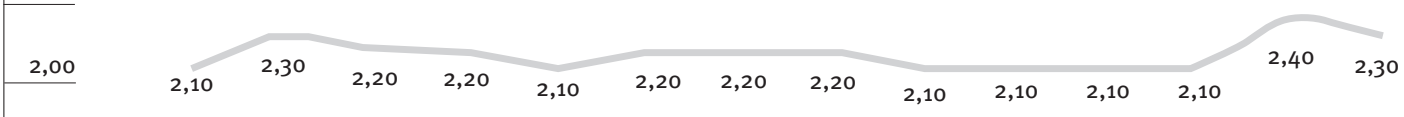

1,50

1,00

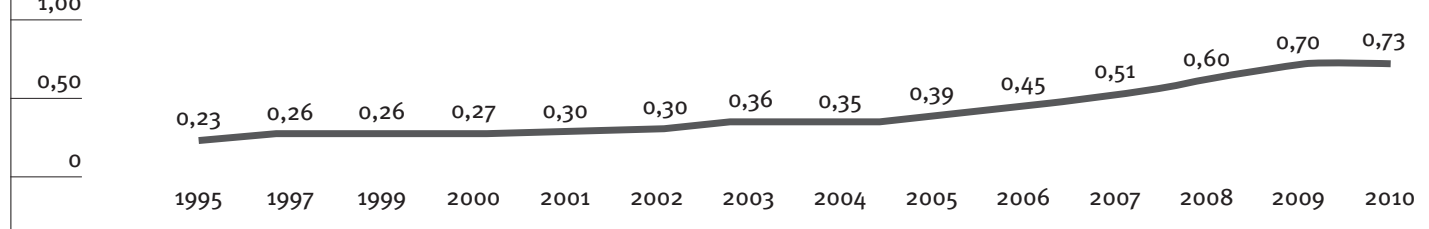

CAPV

UE

Fuente: Elaboración propia a partir de Eurostat (2014) y Eustat (2013).

${ }^{2}$ Estos datos no tienen en cuenta una serie de gastos públicos muy importantes de cara al bienestar de las familias, como el gasto en educación infantil o en desgravaciones fiscales por descendientes. Por otra parte, si se analiza únicamente el gasto correspondiente a la función familia e infancia, no se consideran otras partidas de gasto social, como las rentas mínimas o las prestaciones por desempleo que, si bien no están específicamente orientadas a las familias con hijos/as, las benefician de forma muy significativa.
3 El gasto en esta función está estrechamente relacionado a los niveles de igualdad de oportunidades y a las posibilidades de movilidad social en Europa (Marrero y Rodríguez, 2011). Estos autores elaboran un índice de desigualdad de oportunidades, diferenciando, a partir de datos de la Encuesta de Condiciones de Vida 2005 (EU-SILC), la desigualdad que cabe considerar heredada de la que se deriva del esfuerzo personal. Los datos de los autores ponen de relieve, por una parte, que España contaba en 2005 con un nivel de desigualdad de oportunidades superior a la media europea y, por otra, que el nivel de desigualdad de oportunidades de cada país se relaciona de forma estadísticamente significativa con el gasto público en las funciones de familia e infancia, salud y exclusión social, entre otros aspectos. 
Gráfico 2. Evolución del diferencial de la CAPV respecto a la UE* respecto al gasto en familia en relación al PIB, el gasto en prestaciones sociales en relación al PIB y el gasto en familia en relación al gasto total en prestaciones sociales (1995-2010)

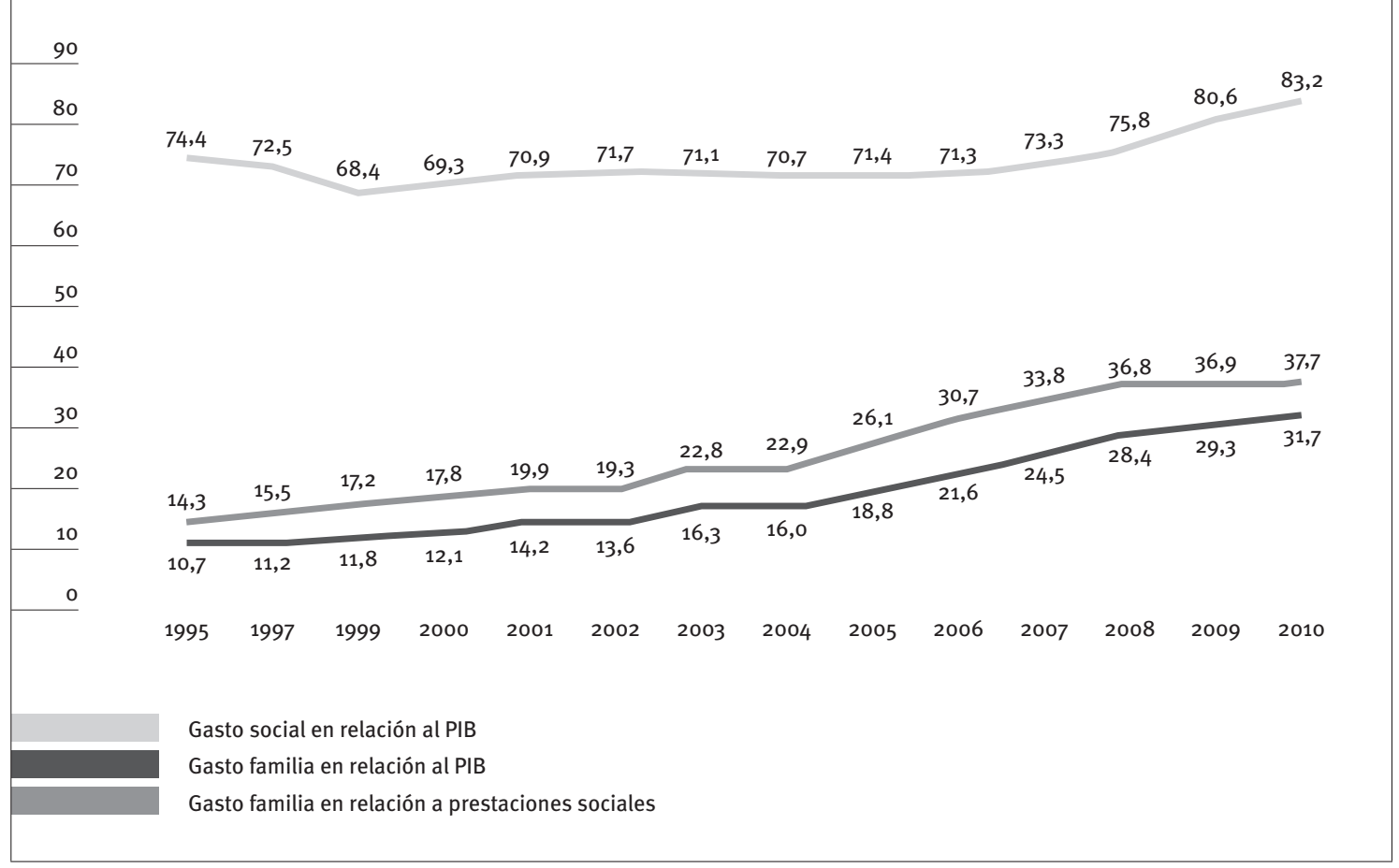

$\star U E=100$.

Fuente: Elaboración propia a partir de Eurostat (2014) y Eustat (2013).

de ingresos y por desempleo). En lo que se refiere, sin embargo, al gasto en la función familia e infancia, si bien el déficit con Europa se ha ido reduciendo en el tiempo, las diferencias son muy elevadas, tanto si se relaciona con el PIB como si se relaciona con el conjunto del gasto en protección social (el gasto que las diferentes administraciones realizan en Euskadi en 2010 en la función familia e infancia en relación al PIB equivalía al $31 \%$ del gasto europeo; el porcentaje que estas prestaciones representan en relación a todo el gasto en protección social en Euskadi equivalía al $37 \%$ de la media europea).

Ello nos lleva a confirmar los datos que ya se ponían de manifiesto en anteriores análisis (SIIS, 2012): el diferencial de la CAPV con Europa en lo que se refiere a las políticas familiares y de apoyo a la infancia se debe no tanto a que en la CAPV se gaste menos en protección social (que también), sino a que el gasto en protección social que se realiza en Euskadi está mucho menos orientado que en los demás países de la UE15 a la protección de las familias con hijos/as a cargo. Así pues, la cuestión relevante es que Euskadi no sólo gasta menos que la media de la UE en protección social, sino que en el ámbito de la familia la diferencia con la UE es todavía más acusada: efectivamente, si Euskadi destina a todas las prestaciones de protección social -salvo a desempleo y supervivientes- una proporción del PIB menor que el conjunto de la UE, es precisamente en la función de familia, junto con la de vivienda, donde esa diferencia es mayor.

\section{Composición del gasto social destinado} a la familia y la infancia en Euskadi y en la OCDE: el peso de las desgravaciones fiscales

Como se ha señalado antes, y como se explica en mayor detalle en trabajos anteriores (SIIS, 2012), los datos que arroja el sistema SEEPROS presentan algunas limitaciones para analizar el conjunto del gasto social que se realiza en los países de la UE y que se relacionan con el bienestar de las familias con hijos/as. Por ello, y siguiendo la misma metodología y fuentes utilizadas en esos trabajos, se ha calculado el gasto público realizado en 2009 en la CAPV y los países de la OCDE en las tres principales partidas de gasto que este organismo contempla en el ámbito de las políticas familiares: las prestaciones económicas, los servicios de atención directa -incluidos los servicios de atención infantil-y las desgravaciones o deducciones fiscales.

En cuanto a la metodología utilizada, los datos correspondientes a los países de la OCDE se han obtenido de la base de datos en línea que publica este organismo. Para la CAPV, el gasto en prestaciones económicas se ha obtenido de la Cuenta de la Protección Social de Eustat (función familia e infancia), mientras que el gasto en servicios se ha calculado sumando el gasto en esa partida que recoge la Cuenta de la Protección Social y el gasto público en centros de educación infantil que recoge la Cuenta de 


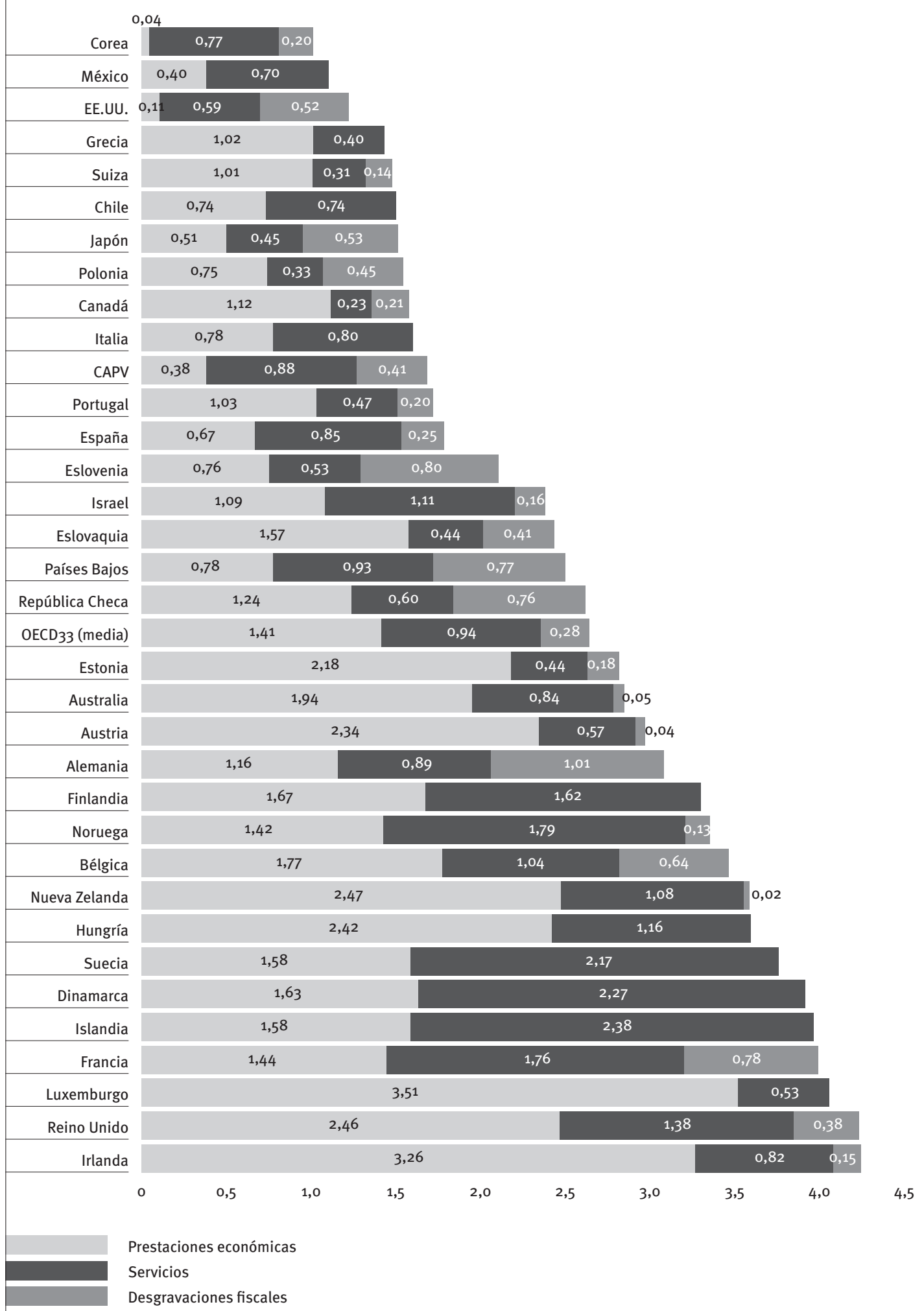

Fuente: Elaboración propia a partir de OCDE (2012), Eustat (2011) y Órgano de Coordinación Tributaria de Euskadi (2009). 
la Educación, también de Eustat. Por último, el gasto en deducciones fiscales corresponde al gasto fiscal computado como deducciones por descendientes en el informe integrado de la Hacienda Vasca correspondiente a $2009^{4}$ (Órgano de Coordinación Tributaria de Euskadi, 2009).

El Gráfico 3 recoge, para todos los países de la OCDE y para la CAPV, el nivel de gasto en esas prestaciones en relación al PIB. Como se puede observar, al igual que el Estado español, la CAPV se encontraría a una distancia considerable del gasto medio de la OCDE $(1,67 \%$, frente al $2,6 \%$ en el conjunto de los países analizados), mientras que una docena de países -entre ellos, nueve de los pertenecientes a la UE15destinan más del $3 \%$ de su PIB a esas prestaciones y servicios. Estas diferencias en términos de gasto agregado se reflejan, como más adelante veremos, en el nivel de protección de las familias vascas, especialmente de aquellas que no reciben prestaciones de garantía de ingresos.

Junto al escaso volumen de gasto total en relación al PIB, una de las particularidades del gasto vasco en familia, cuando se tienen también en cuenta los servicios de atención infantil y las desgravaciones fiscales, es el importante peso que estas últimas juegan en el conjunto del gasto. En efecto, apenas media docena de países recurren en mayor proporción, a la hora de configurar su paquete de apoyo a las familias, a las desgravaciones fiscales (Estados Unidos, Eslovenia, Japón, Alemania, Países Bajos, Polonia y la República Checa). El principal inconveniente de un peso excesivo de los gastos fiscales en el conjunto del gasto en las políticas de apoyo a las familias radica en el hecho de que las familias con menos ingresos se benefician en menor medida de estas deducciones y desgravaciones, dado que en ningún caso éstas superan las cantidades retenidas a cuenta por la hacienda pública, y que tienen como teórico- la cuota líquida o deuda que anualmente se genera con la hacienda pública. límite máximo -independientemente de su montante

Tabla 1. Distribución del gasto en prestaciones económicas, servicios y desgravaciones fiscales orientadas a la infancia. OCDE y CAPV, 2009 (\%)

\begin{tabular}{|c|c|c|c|}
\hline & $\begin{array}{c}\text { Prestaciones } \\
\text { económicas }\end{array}$ & Servicios & Desgravaciones \\
\hline Estados Unidos & 9,02 & 48,36 & 42,62 \\
\hline Eslovenia & 36,19 & 25,24 & 38,10 \\
\hline Japón & 34,46 & 30,41 & 35,81 \\
\hline Alemania & 37,79 & 28,99 & 32,90 \\
\hline Países Bajos & 31,45 & 37,50 & 31,05 \\
\hline Polonia & 49,02 & 21,57 & 29,41 \\
\hline
\end{tabular}

${ }^{4}$ Dada la variedad de fuentes, esta comparación debe considerarse meramente orientativa. En ese sentido, es necesario tener en cuenta que el gasto en servicios de atención directa de la Cuenta de la Protección Social de Eustat ya contempla el gasto en guarderías, por lo que, al sumarse el gasto en atención infantil de la Cuenta de la Educación, ese gasto podría estar siendo sobredimensionado. Para evitar ese efecto, y no disponiéndose del gasto en guarderías correspondiente a 2009 , se ha restado la cantidad destinada a esa partida en 2008. Por otro lado, el Informe Anual Integrado de la Hacienda Vasca (Órgano de Coordinación Tributaria de Euskadi, 2009) tiene en cuenta el total de las deducciones por descendientes a las que los/as contribuyentes vascos tendrían teóricamente derecho. Sin embargo, en la medida en que esas desgravaciones (por sí mismas o sumadas al resto de deducciones) podrían en algunos casos ser superiores a la cuota líquida correspondiente a esas declaraciones, cabe pensar que no todo ese gasto fiscal se realiza de forma efectiva.

\begin{tabular}{|l|c|c|c|}
\hline República Checa & 47,69 & 23,08 & 29,23 \\
\hline CAPV & $\mathbf{2 3 , 0 1}$ & $\mathbf{5 2 , 4 9}$ & $\mathbf{2 4 , 5 1}$ \\
\hline Corea & 3,96 & 76,24 & 19,80 \\
\hline Francia & 36,18 & 44,22 & 19,60 \\
\hline Bélgica & 51,30 & 30,14 & 18,55 \\
\hline Eslovaquia & 64,61 & 18,11 & 16,87 \\
\hline España & 37,83 & 48,29 & 13,89 \\
\hline Canadá & 72,26 & 14,84 & 13,55 \\
\hline Portugal & 60,23 & 27,49 & 11,70 \\
\hline OECD33 (media) & 54,02 & 36,02 & $\mathbf{1 0 , 7 3}$ \\
\hline Suiza & 69,18 & 21,23 & 9,59 \\
\hline Reino Unido & 58,29 & 32,70 & 9,00 \\
\hline Israel & 45,99 & 46,84 & 6,75 \\
\hline Estonia & 78,14 & 15,77 & 6,45 \\
\hline Noruega & 42,51 & 53,59 & 3,89 \\
\hline Irlanda & 76,89 & 19,34 & 3,54 \\
\hline Australia & 68,55 & 29,68 & 1,77 \\
\hline Austria & 79,32 & 19,32 & 1,36 \\
\hline Nueva Zelanda & 69,38 & 30,34 & 0,56 \\
\hline Luxemburgo & 86,88 & 13,12 & 0,00 \\
\hline Islandia & 39,90 & 60,10 & 0,00 \\
\hline Dinamarca & 41,79 & 58,21 & 0,00 \\
\hline Suecia & 42,13 & 57,87 & 0,00 \\
\hline Hungría & 67,60 & 32,40 & 0,00 \\
\hline Finlandia & 50,76 & 49,24 & 0,00 \\
\hline Italia & 49,37 & 50,63 & 0,00 \\
\hline Chile & 50,34 & 50,34 & 0,00 \\
\hline Grecia & 71,33 & 27,97 & 0,00 \\
\hline México & 36,04 & 63,06 & 0,00 \\
\hline & & & \\
\hline
\end{tabular}

Fuente: Elaboración propia a partir de OCDE (2012), Eustat (2011) y Órgano de Coordinación Tributaria de Euskadi (2009).

La diferencia entre el modelo vasco y la media de los países de la OCDE se pone de manifiesto en los Gráficos 4 y 5 . En relación al gasto medio de la OCDE en políticas de familia, el gasto vasco representa apenas un $64 \%$; sin embargo, en el caso de las prestaciones económicas, el gasto vasco sólo llega al $27 \%$ de la media, mientras que en el caso de los servicios de atención directa llega al $93 \%$ y en lo que se refiere a las desgravaciones alcanza el $146 \%$. Desde el punto de vista de la distribución del gasto, mientras que en Euskadi las prestaciones económicas apenas representan el $23 \%$ del gasto en 2009 , en la media del conjunto de los países suponen un $54 \%$. Al contrario, el $52 \%$ del gasto vasco se destina a servicios de atención directa, frente al $36 \%$ de la media; y el $24 \%$, a desgravaciones fiscales, frente al $10 \%$ en la OCDE. 


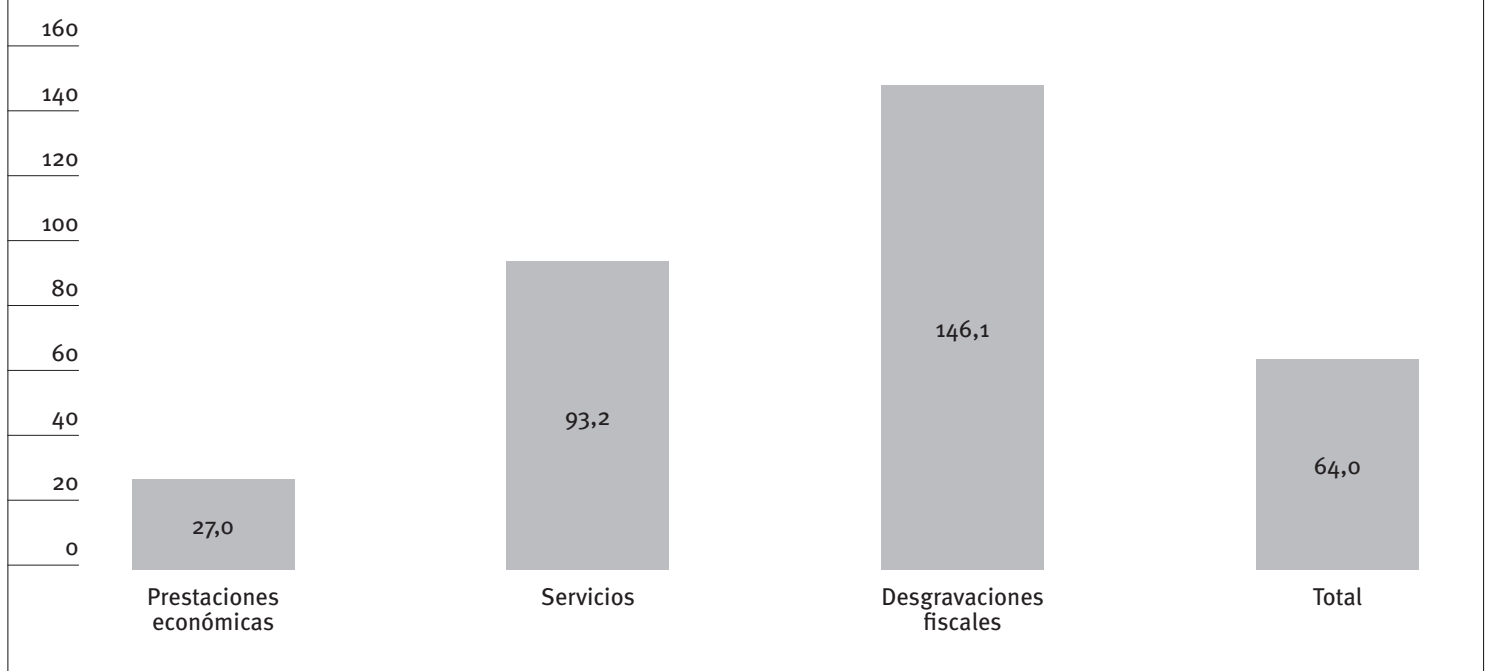

* OCDE $=100$.

Fuente: Elaboración propia a partir de datos de Eustat (2013), Eurostat (2014) y OCDE (2012).

Gráfico 5. Distribución del gasto en políticas de apoyo a las familias. OCDE y CAPV, 2009 (\%)

年

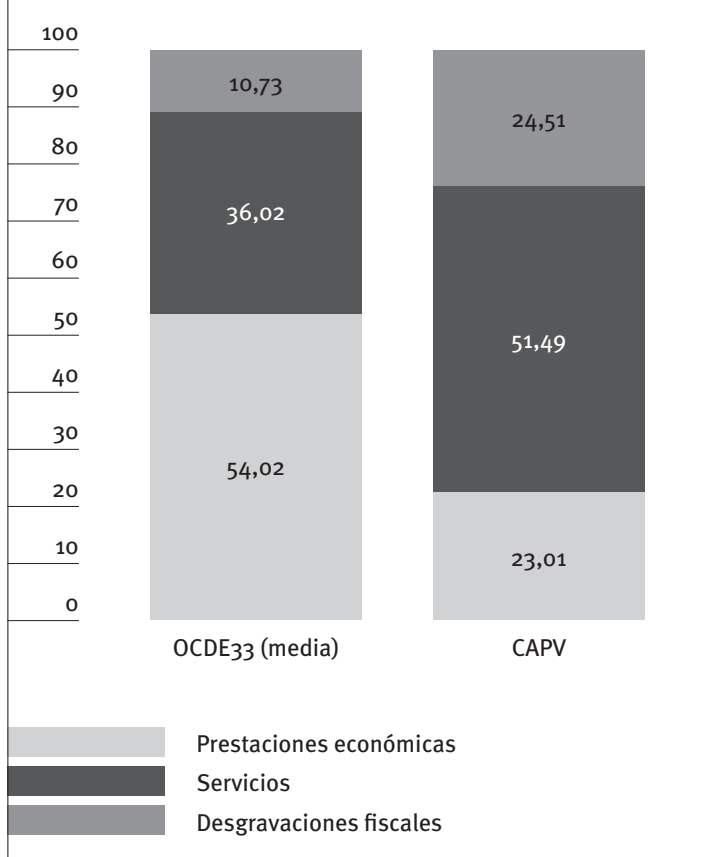

Fuente: Elaboración propia a partir de datos de Eustat (2013), Eurostat (2014) y OCDE (2012).

\section{Una aproximación al método de los paquetes de prestaciones familiares}

\subsection{Aclaraciones metodológicas}

En nuestro entorno más próximo apenas se ha avanzado en el desarrollo de la metodología de los paquetes de ayudas familiares para comparar nuestro modelos de prestaciones familiares con el que existe en Europa, contextualizándolo en un marco político más amplio. Dejando de lado el análisis anterior, basado en datos agregados de gasto, esta metodología se basa en la comparación del montante que recibirían en los diversos países de Europa una serie de familias tipo, que comparten determinadas características básicas en cuanto a renta y composición familiar, de acuerdo a la normativa que rige en sus respectivos países tanto las prestaciones familiares, de vivienda y de garantía de ingresos, como los impuestos sobre la renta y las cotizaciones sociales (Bradshaw y Finch, 2002). Si bien esta aproximación plantea problemas de importancia (la necesidad de centrar el análisis en una serie limitada de familias tipo y, sobre todo, la dificultad de considerar todas las prestaciones disponibles -a escala local, regional y estatal- para cada una de esas familias), lo cierto es que permite analizar con mayor detalle en qué medida familias con necesidades similares obtienen de las administraciones públicas, teóricamente al menos, un tratamiento diferente en los diversos países de Europa.

Aunque no es objeto de este artículo plantear un análisis exhaustivo de la situación de la CAPV respecto a otros países de la UE recurriendo a esta metodología, sí se propone, utilizando la base de datos de prestaciones económicas e impuestos de la OCDE, plantear una primera aproximación a esta cuestión. Para ello, se ha calculado el montante que tres tipos de familias percibirían anualmente en la CAPV en concepto de prestaciones familiares, ayudas a la vivienda y prestaciones de garantía de ingresos, así como el que abonarían en concepto de IRPF y de cotizaciones a la Seguridad Social. Los tres tipos familiares elegidos son:

- La familia de tipo 1 está formada por un padre que percibe el salario medio de su país, y una madre que percibe el $67 \%$ de ese salario medio. Tienen 
dos niños/as, el menor de los cuales tiene 6 años. Cabe considerar a esta familia como una unidad familiar de ingresos medios.

- La familia de tipo 2 está formada por un padre que percibe el $50 \%$ del salario medio de ese país, y una madre que no percibe salario alguno. Como en el caso anterior, la pareja tiene dos niños/as, el menor de los cuales tiene 6 años. Cabe considerar a esta familia como una unidad familiar de bajos ingresos.

- La familia de tipo 3 está constituida por una madre sola, con dos hijos/as a cargo, sin empleo y sin posibilidades de acceso a la prestación o subsidio de desempleo.

Para simplificar el análisis, no se han tenido en cuenta otras configuraciones familiares (como las familias numerosas) ni otros grupos de renta (como las familias con rentas elevadas). En algunos casos, la elección se debe a la disponibilidad de datos en la base de datos en línea de la OCDE5, si bien el motivo fundamental es el deseo de recoger las configuraciones familiares que cabe considerar más habituales en nuestro contexto, bajo tres supuestos de renta específicos (media, baja y sin ingresos).

En todo caso, antes de presentar los resultados, es necesario señalar algunos elementos básicos en relación a las simulaciones realizadas:

- Los datos correspondientes a los países de la OCDE se refieren a 2012 y se han obtenido de la base de datos en línea de esa institución.

- Los datos correspondientes a la CAPV se refieren a diversos años y provienen de estimaciones propias, tanto en lo que se refiere al salario medio ${ }^{6}$ como al montante de las prestaciones familiares o de garantía de ingresos ${ }^{7}$ a las que cada familia tiene derecho, la cuota por IRPF 8 o las cotizaciones a la Seguridad Social. Si bien los ingresos 0 pagos procedentes de los sistemas autonómicos y forales se han estimado expresamente para la CAPV, en lo relativo a las prestaciones y gastos correspondientes a los sistemas de ámbito estatal (fundamentalmente, la Seguridad Social, tanto en lo que se refiere a las cotizaciones como a las posibles prestaciones por hijo/a a cargo) se han utilizado para la CAPV los mismos datos que para el conjunto del Estado.

${ }_{5}^{5}$ Para el segundo perceptor de ingresos, la base de datos ofrece un número muy limitado de opciones retributivas.

${ }^{6}$ El salario medio se ha obtenido a partir de la Encuesta Trimestral de Coste Salarial (INE, 2014).

7 Para calcular la prestación por renta de garantía de ingresos (RGI) se ha utilizado la normativa correspondiente a 2012 y se ha considerado que, en caso de generar derecho a la RGI, se genera igualmente derecho a la prestación complementaria de vivienda (PCV), en su cuantía máxima (320 euros mensuales para una unidad familiar con dos hijos/as).

${ }^{8}$ Para el cálculo, se ha utilizado el sistema de simulación del Departamento de Hacienda de la Diputación Foral de Gipuzkoa correspondiente al ejercicio 2013. En el caso de las familias de tipo 1 y 2 , se ha optado por realizar dos declaraciones individuales, en lugar de una conjunta.
- Las ayudas familiares que se tienen en cuenta, dada la composición de estas familias, son las prestaciones periódicas por hijo/a a cargo, y no posibles asignaciones extraordinarias de pago único, ligadas a excedencias laborales o partos múltiples, entre otras.

- Los datos para los países de la OCDE que se analizan corresponden al conjunto de cada Estado y es posible que exista, sobre todo en los países con sistemas locales o regionales de prestaciones sociales y familiares, una cierta infraestimación de las diferentes ayudas a las que puede acceder una familia. En el caso español, la cuantía y las condiciones de acceso a las rentas mínimas de inserción corresponden a las de la Comunidad de Madrid.

- En los tres casos, se considera que no hay derecho a prestaciones por desempleo (bien por estar ambos cónyuges trabajando, bien por no haberse generado ese derecho, bien por haberse agotado), por lo que estas prestaciones no se tienen en cuenta, pero sí las prestaciones sociales o de garantía de ingresos, también las compatibles con la participación laboral, así como las cotizaciones a la Seguridad Social y el impuesto sobre la renta. La consideración de estos dos elementos permite analizar tanto las prestaciones económicas que las familias perciben de las administraciones como la presión fiscal a la que esas familias están sometidas.

- No se tienen en cuenta las prestaciones en especie-servicios educativos, sociales, sanitarios, culturales y de ocio, infraestructuras-que reciben esas familias y que son financiadas, lógicamente, mediante los impuestos señalados 9 . Tampoco se tienen en cuenta el resto de las tasas e impuestos, como el IVA, que las familias abonan además del IRPF.

- Los cálculos se realizan sobre la base de que las familias reciben en la práctica el montante total de las prestaciones a las que tienen derecho. En la realidad, sin embargo, no es infrecuente que algunas familias no accedan a las prestaciones a las que potencialmente tendrían acceso, especialmente si son de baja cuantía, poco conocidas, de tramitación compleja o se consideran estigmatizantes.

\subsection{Resultados}

El primer dato reseñable es que la CAPV se encuentra, junto con el Estado español, entre los países que abonan una menor cantidad en concepto de ayudas familiares a las familias de cualquier nivel de renta o composición:

9 Diversos estudios han señalado el carácter redistributivo del gasto en servicios públicos de atención directa, especialmente en lo que se refiere a la educación obligatoria y la sanidad. Las prestaciones en efectivo, en cualquier caso, resultan en general más redistributivas (Verbist y Matsaganis, 2014). 
- En el caso de las familias del tipo 3, sin ingresos, la ayuda familiar apenas equivale al $2 \%$ del salario medio, frente al $15 \%$ que, por término medio (no ponderado), corresponde a las familias de estas características en el conjunto de los ámbitos territoriales analizados.

- Las familias de tipo 2, de salarios bajos, pueden percibir una prestación familiar en el Estado (equivalente a 582 euros anuales o el 2,2\% del salario medio español), pero no en la $\mathrm{CAPV}^{10}$. En nuestra comunidad autónoma, estas familias no acceden a ninguna prestación familiar de carácter periódico. Sólo en EE.UU. se da también esta situación.

- En el caso de las familias de renta media (tipo 1), ni en España ni en Euskadi perciben prestación familiar alguna, frente a quince países en los que sí reciben algún tipo de ayuda familiar, generalmente de carácter universal. En estos países, la prestación media equivale al $7,5 \%$ del salario medio.

Tabla 2. Montante anual de las ayudas familiares, por países y tipos de unidades familiares. OCDE y CAPV (\% respecto al salario medio)

\begin{tabular}{|c|c|c|c|}
\hline & Tipo 1 & Tipo 2 & Tipo 3 \\
\hline Irlanda & 10,3 & 10,3 & 49,9 \\
\hline Australia & 0,0 & 19,1 & 42,3 \\
\hline Malta & 3,4 & 8,7 & 39,5 \\
\hline Lituania & 0,0 & 5,0 & 23,6 \\
\hline Canadá & 5,7 & 21,7 & 22,3 \\
\hline Reino Unido & 5,0 & 22,0 & 22,0 \\
\hline Dinamarca & 6,9 & 6,9 & 18,3 \\
\hline Finlandia & 6,3 & 6,3 & 17,6 \\
\hline Suecia & 7,0 & 7,0 & 14,8 \\
\hline Noruega & 4,6 & 4,6 & 13,3 \\
\hline Estados Unidos & 0,0 & 0,0 & 12,3 \\
\hline Islandia & 2,5 & 7,8 & 12,2 \\
\hline Bélgica & 7,2 & 7,2 & 11,9 \\
\hline Austria & 10,7 & 10,7 & 10,7 \\
\hline Alemania & 10,0 & 17,8 & 10,2 \\
\hline Bulgaria & 9,4 & 9,4 & 9,4 \\
\hline Rumanía & 4,0 & 6,4 & 8,8 \\
\hline Grecia & 33,4 & 10,0 & 7,4 \\
\hline Países Bajos & 3,6 & 6,7 & 6,7 \\
\hline Portugal & 0,0 & 5,2 & 6,2 \\
\hline Francia & 4,2 & 4,2 & 4,2 \\
\hline Letonia & 3,0 & 3,0 & 3,0 \\
\hline España & 0,0 & 2,2 & 2,2 \\
\hline CAPV & 0,0 & 0,0 & 2,0 \\
\hline Suiza & 5,5 & 5,5 & 0,0 \\
\hline Italia & 2,9 & 10,6 & 0,0 \\
\hline Media* & 7,5 & 9,0 & 15,4 \\
\hline
\end{tabular}

* La media no tiene en cuenta los países que no abonan prestaciones.

Fuente: Elaboración propia a partir de OCDE (2012) y Eustat (2013).

${ }^{10}$ La razón estriba en que, pese a tratarse de una prestación estatal, se puede acceder a ella con ingresos equivalentes al $50 \%$ del salario medio español, pero no al $50 \%$ del salario medio para la CAPV, que se ha utilizado como referencia y que es sensiblemente más elevado.
Las ayudas que reciben las familias -en Europa y en la CAPV - no se limitan, en cualquier caso, a las prestaciones familiares. La Tabla 3 recoge, para cada composición familiar, la relación entre el montante total de las ayudas percibidas (sumando las ayudas familiares, de vivienda y de ingresos mínimos) y el salario medio de cada país.

Tabla 3. Prestaciones sociales, familiares y de vivienda, por países y tipos de unidades familiares. OCDE y CAPV (\% respecto al salario medio)

\begin{tabular}{|l|c|c|c|}
\cline { 2 - 4 } \multicolumn{1}{c|}{} & Tipo 1 & Tipo 2 & Tipo 3 \\
\hline Lituania & 0,0 & 24,0 & 65,5 \\
\hline Irlanda & 10,3 & 38,9 & 64,8 \\
\hline Reino Unido & 5,0 & 32,0 & 52,6 \\
\hline CAPV & $\mathbf{0 , 0}$ & 18,8 & $\mathbf{5 2 , 0}$ \\
\hline Islandia & 2,5 & 14,9 & 51,4 \\
\hline Finlandia & 13,3 & 26,0 & 50,7 \\
\hline Austria & 10,7 & 18,7 & 49,3 \\
\hline Australia & 0,0 & 27,4 & 47,3 \\
\hline Canadá & 5,7 & 22,8 & 47,3 \\
\hline Noruega & 4,6 & 13,9 & 46,9 \\
\hline Países Bajos & 8,1 & 14,3 & 45,0 \\
\hline Suiza & 5,5 & 5,5 & 44,9 \\
\hline Malta & 3,4 & 13,5 & 44,7 \\
\hline Suecia & 17,2 & 15,7 & 43,6 \\
\hline Alemania & 10,0 & 24,1 & 42,6 \\
\hline Letonia & 3,0 & 5,4 & 41,3 \\
\hline Bélgica & 7,2 & 7,2 & 39,2 \\
\hline Francia & 4,2 & 19,5 & 38,4 \\
\hline Dinamarca & 6,9 & 55,8 & 28,4 \\
\hline Bulgaria & 9,4 & 9,4 & 27,7 \\
\hline Portugal & 0,0 & 5,2 & 27,5 \\
\hline España & 0,0 & 17,2 & 25,5 \\
\hline Estados Unidos & 4,0 & 6,4 & 14,9 \\
\hline Rumanía & 33,4 & 10,0 & 7,4 \\
\hline Grecia & 10,6 & 0,0 \\
\hline Italia & 2,6 & 39,4 \\
\hline Media & & & \\
\hline
\end{tabular}

Fuente: Elaboración propia a partir de OCDE (2012) y Eustat (2013).

Como se observa, las familias de ingresos medios vascas son las únicas -junto con las de España, Lituania, Australia, Portugal y Estados Unidos- que no perciben prestación alguna. Por término medio, los países analizados -sumando las prestaciones familiares, de vivienda y de garantía de ingresosofrecen a estas familias una suma equivalente al $6,4 \%$ del salario medio de cada país.

La situación cambia para la CAPV si tenemos en cuenta a las familias con ingresos salariales bajos. En ese caso, la situación del Estado español sigue siendo muy deficitaria, en la medida en que estas familias sólo tendrían acceso a la prestación por hijo/a a cargo de la Seguridad Social, que equivaldría, como antes se ha señalado, al 2,2 \% del salario medio (se trata de la cuantía relativa más baja de todas las analizadas). Sin embargo, la situación de la CAPV puede considerarse intermedia, puesto que la suma de las ayudas recibidas por una familia de estas características equivaldría 
al 18,8\% del salario medio que se está utilizando como indicador. Se trata de una cantidad algo superior a la media (no ponderada) de los países analizados; se sitúa por debajo de países como Irlanda, Reino Unido o Alemania, pero en la línea de Francia y por encima de Bélgica o Italia. Es importante destacar, sin embargo, que la mejor situación de estas familias en la CAPV no se debe a las políticas familiares -a las que esta composición familiar no tendría derecho, como antes se ha señalado-, sino al sistema de garantía de ingresos y, más concretamente, a la percepción de estímulos al empleo y de la prestación complementaria de vivienda (PCV), que sólo se recibe en caso de vivir en régimen de alquiler.

La situación es, en términos comparativos, aún más favorable para las familias monoparentales sin ingresos salariales, ya que perciben una cantidad cercana al $54 \%$ del salario medio de la CAPV, sólo por debajo de Lituania, Reino Unido e Irlanda. También en este caso, la diferencia se debe fundamentalmente a la renta de garantía de ingresos (RGI) y a la PCV; en ese sentido, es de destacar que en el Estado español (para el que se usa como referencia, como se ha señalado anteriormente, la renta mínima de inserción madrileña), la cobertura total para estas familias apenas equivale al $26 \%$ del ingreso medio, una de las proporciones más bajas de todos los países analizados.

El modelo vasco de atención a las familias de rentas bajas con hijos/as es, por tanto, diferente del que existe en la mayor parte de los países analizados: en la Tabla 4, se observa que, incluso entre las familias con ingresos bajos, las ayudas familiares constituyen la prestación más importante en la mayor parte de los países (en muchos casos se trata, de hecho, de la única ayuda a la que se tienen derecho con esa composición y nivel de renta). En Euskadi, por el contrario, las ayudas provienen del sistema de rentas mínimas, bien mediante la PCV, bien mediante la RGI, modalidad de complemento de ingresos salariales ${ }^{11}$. Algo parecido ocurre entre las familias sin ingresos, aunque, lógicamente, en este caso las prestaciones de asistencia social o garantía de ingresos tienen un peso mayor. Con todo, en siete de los países analizados la mayor parte del paquete de prestaciones que reciben las familias sin ingresos proviene de las ayudas familiares y no del sistema de garantía de ingresos.

Tabla 4. Distribución de las prestaciones sociales, familiares o de vivienda, por países y tipología de ayudas. OCDE y CAPV (\%)

\begin{tabular}{|c|c|c|c|c|c|c|c|c|c|c|c|c|}
\hline & \multicolumn{4}{|c|}{ Tipo 1} & \multicolumn{4}{|c|}{ Tipo 2} & \multicolumn{4}{|c|}{ Tipo 3} \\
\hline & A & B & C & D & A & B & C & D & A & B & C & D \\
\hline Australia & - & - & - & - & 11,9 & 18,3 & 69,8 & 0,0 & 0,0 & 10,6 & 89,4 & 0,0 \\
\hline Austria & 0,0 & 0,0 & 100,0 & 0,0 & 1,3 & 41,3 & 57,4 & 0,0 & 60,2 & 18,1 & 21,7 & 0,0 \\
\hline Bélgica & 0,0 & 0,0 & 100,0 & 0,0 & 0,0 & 0,0 & 100,0 & 0,0 & 69,8 & 0,0 & 30,2 & 0,0 \\
\hline Canadá & 0,0 & 0,0 & 100,0 & 0,0 & 0,0 & 0,0 & 95,1 & 4,9 & 53,0 & 0,0 & 47,0 & 0,0 \\
\hline Dinamarca & 0,0 & 0,0 & 100,0 & 0,0 & 81,8 & 5,8 & 12,3 & 0,0 & 0,0 & 35,5 & 64,5 & 0,0 \\
\hline Finlandia & 0,0 & 0,0 & 47,6 & 52,4 & 20,8 & 39,3 & 24,3 & 15,6 & 33,6 & 31,6 & 34,8 & 0,0 \\
\hline Francia & 0,0 & 0,0 & 100,0 & 0,0 & 44,5 & 33,9 & 21,6 & 0,0 & 52,5 & 36,5 & 11,0 & 0,0 \\
\hline Alemania & 0,0 & 0,0 & 100,0 & 0,0 & 0,0 & 26,3 & 73,7 & 0,0 & 47,6 & 28,5 & 23,9 & 0,0 \\
\hline Grecia & 0,0 & 0,0 & 100,0 & 0,0 & 0,0 & 0,0 & 100,0 & 0,0 & 0,0 & 0,0 & 100,0 & 0,0 \\
\hline Islandia & 0,0 & 0,0 & 100,0 & 0,0 & 0,0 & 47,9 & 52,1 & 0,0 & 60,0 & 16,2 & 23,8 & 0,0 \\
\hline Irlanda & 0,0 & 0,0 & 100,0 & 0,0 & 0,0 & 0,0 & 26,5 & 73,5 & 0,0 & 23,0 & 77,0 & 0,0 \\
\hline Italia & 0,0 & 0,0 & 100,0 & 0,0 & 0,0 & 0,0 & 100,0 & 0,0 & - & - & - & - \\
\hline Países Bajos & 0,0 & 0,0 & 44,3 & 55,7 & 0,0 & 52,9 & 47,1 & 0,0 & 68,2 & 16,9 & 15,0 & 0,0 \\
\hline Noruega & 0,0 & 0,0 & 100,0 & 0,0 & 39,3 & 27,5 & 33,2 & 0,0 & 36,8 & 34,8 & 28,4 & 0,0 \\
\hline Portugal & - & - & - & - & 0,0 & 0,0 & 100,0 & 0,0 & 77,5 & 0,0 & 22,5 & 0,0 \\
\hline CAPV & - & - & - & - & 0,0 & 71,4 & 0,0 & 28,6 & 70,1 & 25,9 & 3,9 & 0,0 \\
\hline España & - & - & - & - & 0,0 & 0,0 & 100,0 & 0,0 & 91,7 & 0,0 & 8,3 & 0,0 \\
\hline Suecia & 0,0 & 0,0 & 40,5 & 59,5 & 0,0 & 33,4 & 44,2 & 22,3 & 28,5 & 37,5 & 34,0 & 0,0 \\
\hline Suiza & 0,0 & 0,0 & 100,0 & 0,0 & 0,0 & 0,0 & 100,0 & 0,0 & 55,4 & 44,6 & 0,0 & 0,0 \\
\hline Reino Unido & 0,0 & 0,0 & 100,0 & 0,0 & 0,0 & 29,9 & 68,8 & 1,3 & 20,1 & 38,0 & 41,9 & 0,0 \\
\hline Estados Unidos & - & - & - & - & 37,4 & 0,0 & 0,0 & 62,6 & 51,7 & 0,0 & 48,3 & 0,0 \\
\hline Bulgaria & 0,0 & 0,0 & 100,0 & 0,0 & 0,0 & 0,0 & 100,0 & 0,0 & 66,2 & 0,0 & 33,8 & 0,0 \\
\hline Letonia & 0,0 & 0,0 & 100,0 & 0,0 & 0,0 & 43,9 & 56,1 & 0,0 & 52,4 & 40,3 & 7,3 & 0,0 \\
\hline Lituania & - & - & - & - & 79,3 & 0,0 & 20,7 & 0,0 & 63,9 & 0,0 & 36,1 & 0,0 \\
\hline Malta & 0,0 & 0,0 & 100,0 & 0,0 & 0,0 & 36,1 & 63,9 & 0,0 & 0,0 & 11,6 & 88,4 & 0,0 \\
\hline Rumanía & 0,0 & 0,0 & 100,0 & 0,0 & 0,0 & 0,0 & 100,0 & 0,0 & 41,2 & 0,0 & 58,8 & 0,0 \\
\hline
\end{tabular}

A: asistencia social o garantía de ingresos. B: ayudas de vivienda. C: ayudas a la familia. D: prestaciones económicas asociadas a la participación en el mercado laboral (in work benefits).

Fuente: Elaboración propia a partir de OCDE (2012) y Eustat (2013). 
Una de las ventajas de la metodología de los paquetes familiares es que permite comparar no sólo el montante de las ayudas recibidas por las diferentes familias, en términos absolutos o en relación al salario, sino también lo que esas familias abonan en concepto de IRPF y de cotizaciones a la Seguridad Social. Para terminar este análisis, la Tabla 5 recoge, precisamente, el nivel de tributación al que están sometidos (montante de pagos por IRPF y cotizaciones a la Seguridad Social en relación al salario bruto inicial) los dos tipos de familia con ingresos propios, así como la variación de sus ingresos iniciales una vez percibidas las ayudas a las que tienen derecho y abonados los impuestos sobre la renta y las cotizaciones correspondientes.

Tabla 5. Nivel de tributación y variación de los ingresos después de transferencias e impuestos para diversas composiciones familiares. OCDE y CAPV (\%)

\begin{tabular}{|l|c|c|c|c|}
\cline { 2 - 5 } \multicolumn{1}{c|}{} & \multicolumn{2}{c|}{ Presión fiscal } & \multicolumn{2}{c|}{ Variación de ingresos* } \\
\cline { 2 - 5 } \multicolumn{1}{c|}{} & Tipo 1 & Tipo 2 & Tipo 1 & Tipo 2 \\
\hline Irlanda & 15,36 & 2,8 & 90,8 & 175,1 \\
\hline Dinamarca & 38,06 & 61,0 & 66,1 & 150,6 \\
\hline Canadá & 20,54 & $-3,2$ & 82,9 & 148,8 \\
\hline Australia & 20,49 & 8,1 & 79,5 & 146,6 \\
\hline Reino Unido & 23,30 & 17,5 & 79,7 & 146,5 \\
\hline Estados Unidos & 17,27 & $-0,6$ & 82,7 & 135,1 \\
\hline Lituania & 20,30 & 13,7 & 79,7 & 134,3 \\
\hline CAPV & 17,52 & 6,5 & $\mathbf{8 2 , 5}$ & 131,2 \\
\hline Alemania & 37,05 & 21,1 & 68,9 & 127,2 \\
\hline Finlandia & 30,83 & 26,0 & 77,1 & 125,9 \\
\hline Islandia & 30,15 & 5,0 & 71,3 & 124,9 \\
\hline Malta & 15,33 & 7,3 & 86,7 & 119,8 \\
\hline Austria & 31,24 & 18,3 & 75,2 & 119,0 \\
\hline Francia & 25,18 & 21,7 & 77,4 & 117,4 \\
\hline Italia & 26,77 & 9,5 & 75,0 & 111,7 \\
\hline Noruega & 27,76 & 17,1 & 75,0 & 110,7 \\
\hline Países Bajos & 33,76 & 21,5 & 71,1 & 107,2 \\
\hline Suecia & 29,91 & 27,0 & 80,4 & 104,5 \\
\hline Bélgica & 38,75 & 10,1 & 65,5 & 104,3 \\
\hline Letonia & 26,71 & 11,0 & 75,1 & 99,8 \\
\hline Portugal & 18,93 & 11,0 & 81,1 & 99,3 \\
\hline Grecia & 31,68 & 21,7 & 88,3 & 98,3 \\
\hline España & 18,85 & 6,4 & 81,1 & 98,1 \\
\hline Bulgaria & 21,61 & 21,6 & 84,0 & 97,1 \\
\hline Rumanía & 27,66 & 21,6 & 74,7 & 91,2 \\
\hline Suiza & 28,47 & 27,3 & 74,8 & 83,6 \\
\hline Media & 25,90 & 15,9 & 77,9 & 119,4 \\
\hline & & & & \\
\hline
\end{tabular}

* Tras impuestos y prestaciones.

Fuente: Elaboración propia a partir de OCDE (2012) y Eustat (2013).

Los datos de la tabla confirman la escasa presión fiscal vasca: la familia de tipo 1 abonaría en concepto de IRPF y de cotizaciones a la Seguridad Social el $17 \%$ de sus ingresos originales, frente a una media no ponderada del $25,9 \%$ en el conjunto de los países analizados ${ }^{12}$; sólo Irlanda, Estados Unidos y Malta tie-

${ }^{12}$ El modelo no considera las posibles deducciones a las que las familias tendrían derecho por inversión o alquiler en vivienda. En caso de haberlo hecho, la presión fiscal efectiva habría sido aún menor. nen una presión más baja para este tipo de familias. En el caso de las familias de renta baja, la situación es similar, y la diferencia respecto a la tributación media es incluso mayor.

Tras el pago de los impuestos correspondientes y la percepción de las prestaciones económicas a las que tienen derecho, las familias vascas con rentas medias mantienen el $82 \%$ de sus ingresos originales, cinco puntos por encima de la media. Por el contrario, Euskadi es uno de los países donde la renta inicial de las familias con rentas bajas más se incrementa tras la percepción de ayudas y el pago de impuestos (hasta el $131 \%$ de la renta original). Letonia, Portugal, Grecia, España, Bulgaria, Rumanía y Suiza son, por el contrario, los únicos países donde la situación de las familias de renta baja empeora tras percibir las ayudas y pagar los impuestos correspondientes.

En resumen, el sistema de prestaciones sociales e impuestos existente en la CAPV -determinado tanto por la administración central como por la autonómica y la foral- ofrece a las familias de rentas medias un nivel de protección mucho más bajo que en los demás países de Europa, debido fundamentalmente a la ausencia de un modelo universal de ayudas familiares, si bien esta escasa protección se ve compensada por -o es producto de- una presión fiscal también sensiblemente más baja ${ }^{13}$, debido en parte a la existencia de un sistema de deducciones fiscales por descendientes que fundamentalmente beneficia a las rentas medias $y$ altas ${ }^{14}$. Las familias con ingresos salariales bajos reciben una protección homologable a la que se recibe en otros países de la UE, si bien ese nivel de protección está muy condicionado a la percepción de la RGI en su modalidad de complemento salarial y de la PCV, lo que, en la práctica, no siempre sucede. En caso de no acceder a estas dos ayudas, el nivel de protección al que estas familias tendrían derecho sería muy bajo (así como su presión fiscal, como en el caso anterior). Finalmente, las familias sin ingresos, si bien no se benefician de las deducciones fiscales, reciben una nivel de protección superior incluso al que se recibe en otros países de la UE, debido a la suma de las cuantías de la RGI y la PCV.

El papel que el sistema de rentas mínimas establecido en Euskadi juega en lo que se refiere a la protección de las familias más desfavorecidas -así como sus limitaciones- se ha expuesto en anteriores trabajos (SIIS, 2013a). En este sentido, los datos disponibles muestran el importantísimo efecto que

${ }^{13}$ Como se ha señalado en anteriores trabajos, a la hora de contextualizar las diferencias entre Euskadi y el conjunto de los países de la UE, es necesario tener en cuenta que las políticas familiares europeas tienen una notable dimensión universalista, asociada a unos ingresos públicos muy elevados, que hace difícil comparar esas políticas con lo que se hace en España y en Euskadi, con un gasto mucho más selectivo asociado a ingresos públicos muy inferiores. Es lógico, por tanto, que retorne en nuestro contexto a la ciudadanía, mediante servicios y prestaciones, una parte más reducida del PIB (SIIS, 2012).

${ }^{14}$ En la simulación realizada, las familias con bajos ingresos y declaración conjunta apenas se benefician del $50 \%$ del montante de la deducción a la que teóricamente tienen derecho. Si su renta fuera un $50 \%$ mayor, se habrían beneficiado de la deducción en su integridad. 
ese sistema tiene en la prevención y la reducción de la pobreza infantil, así como su clara orientación familiar. Desde este punto de vista, cabe insistir en que el sistema vasco de garantía de ingresos -articulado en torno a la renta de garantía de ingresos (RGI), la prestación complementaria de vivienda (PCV) y las ayudas de emergencia social (AES)- está jugando un papel esencial en la contención de las situaciones de pobreza infantil en Euskadi ${ }^{15}$.

El papel que la RGI desempeña como política de apoyo a las familias con hijos/as más desfavorecidas plantea, en cualquier caso, inconvenientes que es preciso destacar:

- Se trata, por una parte, como hemos visto, de un sistema relativamente atípico en Europa, donde incluso para las familias con rentas bajas (al menos cuando existe cierta vinculación con el mercado laboral), las ayudas familiares constituyen el componente principal del paquete de ayudas. Cabe pensar que las ayudas familiares -incluso si estén determinadas por el nivel de renta- tienen un carácter más normalizado e inclusivo, y presentan menos problemas de take up o no acceso por parte de las personas potencialmente demandantes. Efectivamente, los datos de la Encuesta de Pobreza y Desigualdades Sociales (Departamento de Empleo y Asuntos Sociales, 2012) evidencian que en torno a un $25 \%$ de las familias potencialmente demandantes de la RGI (en situación de pobreza real) no acceden a esta prestación ${ }^{16}$.

15 En 2012, cerca de 25.000 niños/as menores de 16 años -en torno a un $8 \%$ del total de la población de esa edad- se beneficiaban por esas prestaciones, y algo más del $25 \%$ de las unidades familiares perceptoras (incluidas las formadas únicamente por pensionistas que percibían la RGI en su modalidad de complemento de pensiones) tenían hijos/as a cargo. En octubre de 2012 , se destinaron a familias con hijos/as algo más de 11 millones de euros, que representaban casi el $38 \%$ del gasto total en RGI. Si a esa cantidad se añaden los 2,1 millones mensuales destinados al pago de la PCV, tendríamos un gasto anual estimado de 160 millones de euros dirigido a las familias con hijos e hijas. Como consecuencia de ese esfuerzo económico, el sistema conformado por la RGI, las AES y la PCV reduce en un $30 \%$ las tasas de pobreza infantil en Euskadi. De acuerdo a los datos de la Encuesta de Pobreza y Desigualdades Sociales Encuesta de Pobreza y Desigualdades Sociales (Departamento de Empleo y Asuntos Sociales, 2012) correspondiente a 2012, el $11 \%$ de la población vasca residente en familias con menores de 18 años a cargo estaría en una situación de pobreza real en caso de no existir estas ayudas; cuando se tienen en cuenta tales ayudas, ese porcentaje desciende al 7,6\%. Además, la intensidad de la pobreza -es decir, la distancia entre el umbral de pobreza y los ingresos que obtienen las familias que están por debajo de ese umbral- se reduce en un $69 \%$, lo cual representa una mejora decisiva de las condiciones de vida de esas familias.

${ }^{16}$ De acuerdo a esos datos, la población en familias con hijos/ as en situación de pobreza real se divide en Euskadi en tres grandes grupos. El más pequeño -que representa el $30 \%$ de los casos- está formado por familias originalmente en situación de pobreza real que salen de esa situación gracias, precisamente, a las ayudas recibidas; el $43 \%$ de ese grupo percibe también ayudas del sistema vasco de garantía de ingresos, pero en cuantía insuficiente para superar el umbral de pobreza real (si bien, como es lógico, su situación se ve claramente mejorada debido al uso de estas prestaciones); finalmente, el $26 \%$ de las familias en situación de pobreza real no acceden a estas ayudas, pese a las circunstancias en que se encuentran. Por lo tanto, las ayudas sólo llegarían al $73 \%$ de la población potencialmente demandante, y sólo en el $30 \%$ de los casos resultarían plenamente eficaces (SIIS, 2013b).
- La capacidad de reducción de la pobreza infantil del sistema RGI es menor de lo deseable ${ }^{17}$. En este sentido, la capacidad de reducción de la pobreza del sistema vasco de rentas mínimas es mayor para las familias sin hijos/as que para las familias con hijos/as, lo que puede deberse al propio diseño de las cuantías de la RGI y al sistema establecido para la consideración del tamaño familiar. Desde este punto de vista, si las cuantías de la RGI vasca se comparan con las de otros países de la UE, se comprueba que en ellos la composición familiar se tiende a tomar en cuenta en mayor medida que en Euskadi. Si en el caso de las unidades unipersonales la RGI vasca es completamente homologable a las ayudas de la UE (tanto en lo que se refiere a la cuantía como a la tasas de remplazo de la renta mediana que garantizan), se observa que cuanto más amplio es el tamaño de la familia, más lejos queda la cuantía de la RGI vasca de la cuantía media establecida en los países analizados. En efecto, mientras que en el caso de las unidades unipersonales la cuantía vasca es superior a la media, en el de las familias biparentales con menores resulta sensiblemente inferior a esa media.

\section{Conclusiones}

Las conclusiones de este análisis son, por fuerza, similares a las que se hacían en trabajos anteriores (SIIS, 2012): si el sistema vasco se caracteriza por un gasto reducido en el conjunto de la protección social, el déficit es todavía más acusado en el ámbito de las políticas de apoyo a las familias, que constituyen -en consonancia con el carácter mediterráneo del Estado de bienestar imperante en nuestro país- un ámbito particularmente poco desarrollado. Una segunda característica, desde el punto de vista del gasto, de las políticas de apoyo a las familias en la CAPV, es el peso que en ellas tienen los gastos fiscales, que sin duda benefician especialmente a las familias de rentas medias y altas, en detrimento de las familias con rentas más bajas, cuya deuda con Hacienda es en ocasiones inferior a las deducciones a las que teóricamente tendrían derecho. Ello hace que estas familias no se beneficien, o lo hagan sólo parcialmente, de unas deducciones por descendientes de las que las rentas medias y altas se benefician íntegramente.

En todo caso, el método de los paquetes familiares -utilizado para este trabajo de forma orientativa y preliminar- pone de manifiesto que si bien la situación de las familias vascas de rentas medias es, en relación a las familias europeas con la misma composición y nivel de renta, muy deficitaria -en la medida en que tienen acceso a un nivel de ayudas mucho menor-, las familias con acceso a la RGI -incluso cuando tienen un empleo- reciben un nivel de protección mucho mayor, homologable al que se recibe

${ }^{17}$ Conviene recordar que los cálculos anteriores se han realizado en relación al salario medio de cada país y no a los umbrales de pobreza. 
en otros países de Europa. Este mejor tratamiento se deriva, en cualquier caso, del acceso al sistema de rentas mínimas y no de la percepción de prestaciones familiares, a diferencia de lo que ocurre en la mayor parte de los países de Europa.
Por su parte, la comparativamente peor situación de las familias de ingresos medios se corresponde -0 , más bien, se deriva- de unos niveles de tributación que son también para esas familias mucho más reducidos que los de las familias con el mismo nivel de renta de la mayor parte de los países de Europa.

\section{Bibliografía referenciada}

BRADSHAW, J.; y FINCH, N. A. (2002): Comparison of Child Benefit Packages in 22 Countries, serie Research Report, n-174, Londres, Department for Work and Pensions. [<http://www.eustat.es/estadisticas/tema_94/ opt_o/ti_Cuenta_de_la_Proteccin_Social/ temas.html>].

- (2011): Cuenta de la Educación, Vitoria-Gasteiz, Eusko Jaurlaritza-Gobierno Vasco [<http://www. eustat.es/estadisticas/tema_190/opt_o/ ti_Cuenta_de_la_Educacin/temas.html $/$.

INE (2014): Encuesta Trimestral de Coste Salarial, Madrid, Instituto Nacional de Estadística.

MARRERO, G. A.; y RODRÍGUEZ, J. G. (2011): “Análisis y determinantes de la desigualdad de oportunidades en España y Europa”, en: CABRALES, A.; y CELENTANI, M. (coords.), Talento, esfuerzo y movilidad social,
Madrid, Fundación de Estudios de Economía Aplicada.

OCDE (2012): "Taxes and benefits", en OCDE, OECD Social Expenditure Statistics, Ginebra, Organización para la Cooperación y el Desarrollo Económicos [rhttp://dx.doi.org/10.1787/data-00201-en〉].

ÓRGANO DE COORDINACIÓN TRIBUTARIA DE EUSKADI (2009): Informe Anual Integrado de la Hacienda Vasca, Bilbao, Órgano de Coordinación Tributaria de Euskadi.

SIIS CENTRO DE DOCUMENTACIÓN Y ESTUDIOS (2013a): Fiscalidad, activación y apoyo a las familias: el papel de las deducciones reembolsables. Panorama internacional, Donostia-San Sebastián, Diputación Foral de Gipuzkoa [<http://www.siis.net/documentos/informes/ Fiscalidad.pdf〉].
Pobreza infantil en Euskadi, Vitoria-Gasteiz, Eusko Jaurlaritza-Gobierno Vasco.

- (2012): "Políticas de apoyo a las familias en la Unión Europea y en la CAPV", Zerbitzuan, ํㅜㄴ 52, págs. $63-86$ [<http://dx.doi.org/10.5569/11347147.52.04)].

VERBIST, G.; y MATSAGANIS, M. (2014): "Redistributive capacity of services in the European Union", en CANTILLON, B.; y VANDENBROUCKE, F. (eds.) Reconciling Work and Poverty Reduction. How Successful are European Welfare States?, serie International Policy Exchange Series, Oxford, Oxford University Press. 